\title{
A LOCALLY HYPERBOLIC 3-MANIFOLD THAT IS NOT HOMOTOPY EQUIVALENT TO ANY HYPERBOLIC 3-MANIFOLD
}

\author{
TOMMASO CREMASCHI
}

\begin{abstract}
We construct a locally hyperbolic 3-manifold $M$ such that $\pi_{1}(M)$ has no divisible subgroups. We then show that $M$ is not homotopy equivalent to any complete hyperbolic manifold.
\end{abstract}

\section{INTRODUCTION}

In Cre17. we constructed an example of a locally hyperbolic 3-manifold without divisible elements that was not homeomorphic to any complete hyperbolic 3manifold. This answered a question of Agol DHM06, Mar07. In the present work we show how, using techniques similar to [Cre17, one can construct a locally hyperbolic 3-manifold $M$ without divisible elements such that $M$ is not homotopy equivalent to any complete hyperbolic 3-manifold.

Theorem. There exists a 3-manifold $M$ that is locally hyperbolic without divisible elements in $\pi_{1}(M)$ that is not homotopy equivalent to any hyperbolic 3-manifold.

Notation. We use $\simeq$ for homotopic, and by $\pi_{0}(X)$ we intend the connected components of $X$. With $\Sigma_{g, k}$ we denote the genus $g$ orientable surface with $k$ boundary components. By $N \hookrightarrow M$ we denote embeddings, while $S \uparrow M$ denotes immersions.

\section{BACKGROUND}

We now recall some facts and definitions about the topology of 3-manifolds; more details can be found in [Hem76, Hat07, Jac80.

An orientable 3-manifold $M$ is said to be irreducible if every embedded sphere $\mathbb{S}^{2}$ bounds a 3-ball. A map between manifolds is said to be proper if it sends boundaries to boundaries and if preimages of compact sets are compact. We say that a connected properly immersed surface $S \rightarrow M$ is $\pi_{1}$-injective if the induced map on the fundamental groups is injective. Furthermore, if $S \hookrightarrow M$ is embedded and $\pi_{1}$-injective, we say that it is incompressible. If $S \hookrightarrow M$ is a non- $\pi_{1}$-injective two-sided 1 surface by the Loop Theorem we have that there is a compressing disk $D \hookrightarrow M$ such that $\partial D=D \cap S$ and $\partial D$ is nontrivial in $\pi_{1}(S)$.

An irreducible 3 -manifold $(M, \partial M)$ is said to have incompressible boundary if every map $\left(D^{2}, \partial D^{2}\right) \hookrightarrow(M, \partial M)$ is homotopic via a map of pairs into $\partial M$. Therefore, $(M, \partial M)$ has incompressible boundary if and only if each component $S \in \pi_{0}(S)$

Received by the editors December 24, 2018, and, in revised form, November 12, 2019.

The author gratefully acknowledges support from the U.S. National Science Foundation grant DMS-1564410: Geometric Structures on Higher Teichmüller Spaces.

${ }^{1}$ The normal bundle on $S$ in $M$ is trivial. 
is incompressible, that is, $\pi_{1}$-injective. An orientable, irreducible, and compact 3manifold is called Haken if it contains a two-sided $\pi_{1}$-injective surface. A 3-manifold is said to be acylindrical if every map $\left(S^{1} \times I, \partial\left(S^{1} \times I\right)\right) \rightarrow(M, \partial M)$ can be homotoped into the boundary via maps of pairs.

Definition 1.1. A 3-manifold $M$ is said to be tame if it is homeomorphic to the interior of a compact 3-manifold $\bar{M}$.

Definition 1.2. We say that a codimension zero submanifold $N \stackrel{\iota}{\hookrightarrow} M$ forms a $S$ cott core if the inclusion map $\iota$ is a homotopy equivalence.

By [Sco73, HS96, RS90, given an orientable irreducible 3-manifold $M$ with finitely generated fundamental group, a Scott core exists and is unique up to homeomorphism.

Let $M$ be a tame 3-manifold. Then given a Scott core $C \hookrightarrow M \subseteq \bar{M}$ with incompressible boundary we have that, by Waldhausen's cobordism theorem Wal68, every component of $\bar{M} \backslash C$ is a product submanifold homeomorphic to $S \times I$ for $S \in \pi_{0}(\partial C)$.

Definition 1.3. Given a core $C \hookrightarrow M$ we say that an end $E \subseteq \overline{M \backslash C}$ is tame if it is homeomorphic to $S \times[0, \infty)$ for $S=\partial E$.

A core $C \subseteq M$ gives us a bijective correspondence between the ends of $M$ and the components of $\partial C$. We say that a surface $S \in \pi_{0}(\partial C)$ faces the end $E$ if $E$ is the component of $\overline{M \backslash C}$ with boundary $S$. It is a simple observation that if an end $E$ facing $S$ is exhausted by submanifolds homeomorphic to $S \times I$, then it is a tame end.

Definition 1.4. A 3-manifold $M$ is said to be locally hyperbolic if every cover $N \rightarrow M$ with $\pi_{1}(N)$ finitely generated is hyperbolizable.

Remark 1.5. By the Tamenes:2 Ago98,CG06 and Geometrization Theorems [Thu82, Per03b, Per03c, Per03a this is equivalent to saying that every cover $N \rightarrow M$ with $\pi_{1}(N)$ finitely generated is the interior of a compact, irreducible 3 -manifold that is atoroidal and with infinite $\pi_{1}$.

Definition 1.6. An element $g$ in a group $G$ is said to be divisible if for all $n \in \mathbb{N}$ there exists $\alpha \in G \backslash\{e\}$ such that $g=\alpha^{n}$.

1.1. Homotopy equivalences. We now recall some facts about homotopy equivalences of irreducible 3-manifolds. For details see [Joh79, JS78, CM04].

Definition 1.7. A Seifert fibered 3-manifold $M$ is a compact, orientable, irreducible 3-manifold that has a fibration by circles.

Definition 1.8. Given a 3-manifold $M$ and a manifold $N, \operatorname{dim}(N) \leq 3$, a continuous map $f:(N, \partial N) \rightarrow(M, \partial M)$ is essential if $f_{*}$ is $\pi_{1}$-injective and $f$ is not homotopic via map of pairs to a map $g$ such that $g(T) \subseteq \partial M$.

Definition 1.9. Given an irreducible, compact 3-manifold $(M, \partial M)$ with incompressible boundary, a characteristic submanifold for $M$ is a codimension zero sub-

\footnotetext{
${ }^{2}$ Finite type hyperbolic 3-manifolds are homeomorphic to the interior of compact 3-manifolds.
} 
manifold $(N, R) \hookrightarrow(M, \partial M)$ satisfying the following properties:

(i) every component $(\Sigma, \partial \Sigma) \in \pi_{0}(N)$ is an $I$-bundle or a Seifert fibered manifold;

(ii) $\partial N \cap \partial M=R$;

(iii) all essential maps of a Seifert fibered manifold $S$ into $(M, \partial M)$ are homotopic as maps of pairs into $(N, R)$;

(iv) $N$ is minimal, i.e., there are no two components homotopic to each other.

By work of Johannson and Jaco-Shalen we have such a submanifold for compact, irreducible 3-manifolds with incompressible boundary [CM04 2.9.1], as shown in the theorem below.

Theorem (Existence and uniqueness). Let $(M, \partial M)$ be a compact, irreducible 3manifold with incompressible boundary. Then there exists a characteristic submanifold $(N, R) \hookrightarrow(M, \partial M)$, and any two characteristic submanifolds are isotopic.

This is also called the JSJ or annulus-torus decomposition. One of the main applications of the JSJ decomposition is the following theorem [Joh79, 24.2].

Theorem 1.10. Let $(M, \partial M)$ and $\left(M^{\prime}, \partial M^{\prime}\right)$ be compact irreducible 3-manifolds with incompressible boundary, and denote by $(N, R),\left(N^{\prime}, R^{\prime}\right)$, respectively, their characteristic submanifolds. Given a homotopy equivalence $f: M \rightarrow M^{\prime}$, then we have a map $\phi$ homotopic to $f$ such that:

(i) $\phi: \overline{M \backslash N} \cong \overline{M^{\prime} \backslash N^{\prime}}$ is a homeomorphism;

(ii) $\phi: N \rightarrow N^{\prime}$ is a homotopy equivalence.

In particular, if $M$ is acylindrical we have that $M$ has no characteristic submanifold; therefore, any homotopy equivalence $f: M \rightarrow N$ is homotopic to a homeomorphism.

Definition 1.11. Given an essential properly embedded annulus $(A, \partial A)$ in $(M$, $\partial M)$ a Dehn flip of $M$ along $A$ is the 3-manifold $N$ obtained by cutting $M$ along $A$ picking a homeomorphism $f: M|A \stackrel{\simeq}{\rightarrow} M| A$ that is the identity on $A$ and regluing $f(M \mid A)$ along $f(A)$ either via the identity or via the map $\phi(x, t)=(x, 1-t)$ where we parametrised $A$ by $S^{1} \times[0,1] \cong A$.

A Dehn flip of $M$ along $A$ naturally gives a homotopy equivalence $h: M \rightarrow N$ which we will also denote by a Dehn flip.

Moreover, as a consequence of Theorem 1.10 (see Joh79, JS78,CM04), we have that homotopy equivalences of $M$ are generated by Dehn flips along annuli contained in the boundary of the characteristic submanifold of $M$; see Theorem Joh79, 29.1]. As a consequence of Johansson homotopy equivalence theory for Haken 3manifold we get the following lemma.

Lemma 1.12. If the characteristic submanifold of a Haken 3-manifold $M$ is given by one embedded separating cylinder $C$, then any 3-manifold $N$ homotopy equivalent to $M$ is either homeomorphic to $M$ or to a Dehn flip along $C$.

\section{Construction of the example}

Consider the 3-manifold $A$ obtained as a thickening of the 2-complex given by gluing a genus two surface $S$ and a torus $T$ so that a meridian of $T$ is identified with a separating simple closed curve $\gamma$ of $S$. Note that $\partial A$ is formed by two genus 
two surfaces both of which are incompressible in $A$. Let $B, C$ be two copies of a hyperbolizable, acylindrical 3-manifold with incompressible genus two boundary (for example, see [Thu97, 3.3.12]), and glue $B, C$ to the 3 -manifold $A$, one to each boundary component. Then we obtain a closed 3-manifold $X$ as shown in Figure 1 .

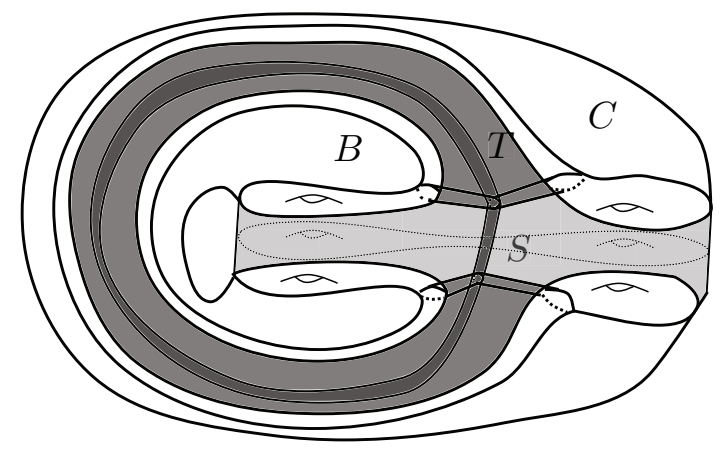

Figure 1. Schematic of the manifold $X$ where the starting manifold $A$ is shaded and the essential torus $T$ is dark grey.

Note that the manifold $X$ is not hyperbolizable since it contains the essential torus $T$ and that the surface $S$ is incompressible and separating in $X$.

Remark 2.1. The 3-manifold $Y \doteq X \mid S=X \backslash N_{\varepsilon}(S)$ is hyperbolizable, with incompressible boundary, and its characteristic submanifold is given by an annulus connecting the two distinct boundaries. Thus, any annulus with both boundary components on the same surface is boundary parallel.

The infinite cyclic cover $M$ of $X$ is obtained by gluing infinitely many copies $\left\{Y_{i}\right\}_{i \in \mathbb{N}}$ of $Y$ along their boundaries, as seen in Figure 2,

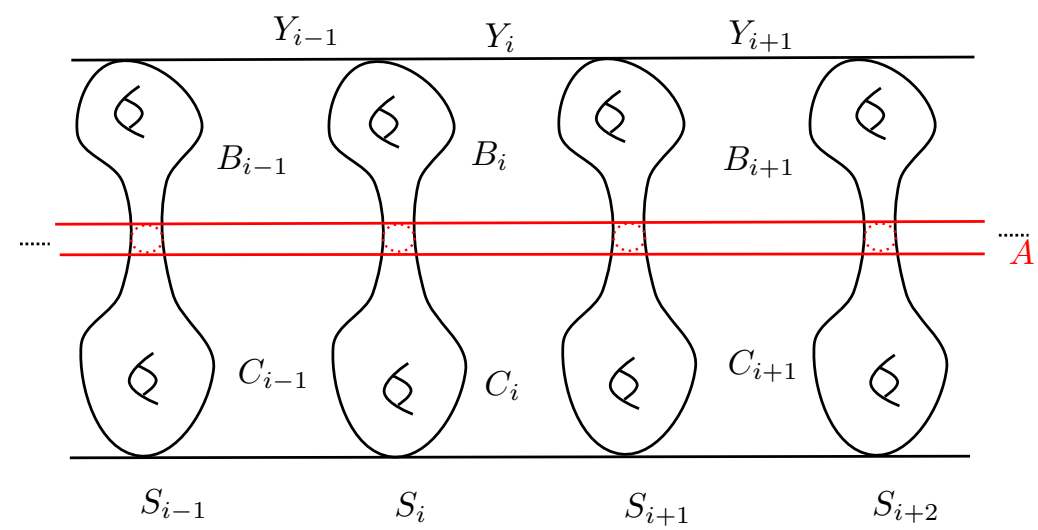

Figure 2 
Denote by $\left\{S_{i}\right\}_{i \in \mathbb{Z}} \subseteq M$ the lifts of $S$. The surfaces $\left\{S_{i}\right\}_{i \in \mathbb{Z}}$ are all genus two and incompressible in $M$. Moreover, we denote by $M_{i, j} \doteq \bigcup_{i \leq k \leq j} Y_{k}$ the compact submanifolds of $M$ co-bounded by $S_{i}, S_{j}$ for $i<j$ and by $A$ the properly embedded annulus in $M$ that is the lift of the essential torus $T$, and we let $A_{i, j} \doteq M_{i, j} \cap A$. With an abuse of notation we denote by $\gamma \in \pi_{1}\left(M_{i, j}\right)$ the elements corresponding to $\pi_{1}(A)$.

In the remainder of this work we will show that the manifold $M$ satisfies the following three properties:

(1) $\pi_{1}(M)$ has no divisible elements;

(2) $M$ is locally hyperbolic;

(3) $M$ is not homotopy equivalent to any hyperbolic 3-manifold.

These will give us the theorem . We will now show that $M$ is locally hyperbolic and that $\pi_{1}(M)$ has no divisible elements.

Lemma 2.2. The manifold $M$ has no divisible elements in $\pi_{1}(M)$.

Proof. The manifold $M$ is the cover of a compact 3-manifold $X$, thus we have that $\pi_{1}(M) \subseteq \pi_{1}(X)$. Since $X$ is irreducible, compact, and with infinite $\pi_{1}$ by [Sha75], we have that $\pi_{1}(X)$ has no divisible elements in $\pi_{1}$.

Lemma 2.3. The manifold $M$ is locally hyperbolic, and for all covers $N_{-i, i}$ corresponding to $\pi_{1}\left(M_{-i, i}\right)$ the homotopy equivalences $\widetilde{\iota}$ : int $\left(M_{-i, i}\right) \hookrightarrow N_{-i, i}$, for $\widetilde{\iota}$ the lift of the inclusion, are homotopic to homeomorphism.

Proof. We first claim that $M$ is atoroidal. Let $\mathbb{T} \subseteq M$ be an essential torus. Since $\mathbb{T}$ is compact it intersects at most finitely many $\left\{S_{i}\right\}_{i \in \mathbb{Z}}$. Moreover, up to an isotopy we can assume that $\mathbb{T}$ is transverse to all $S_{i}$ and that it minimises $\left|\pi_{0}\left(T \cap \bigcup_{i \in \mathbb{Z}} S_{i}\right)\right|$. If $\mathbb{T}$ does not intersect any $S_{i}$ we have that it is contained in a submanifold homeomorphic to $Y$ (see Remark 2.1), which is atoroidal, and so $\mathbb{T}$ is not essential.

Since both the $S_{i}$ 's and $\mathbb{T}$ are incompressible by our minimality condition we have that the components of the intersection $\mathbb{T} \cap S_{i}$ are essential pairwise disjoint simple closed curves in $\mathbb{T}, S_{i}$. Thus, $\mathbb{T}$ is decomposed by $\bigcup_{i \in \mathbb{Z}} \mathbb{T} \cap S_{i}$ into finitely many parallel annuli. Consider $S_{k}$ such that $\mathbb{T} \cap S_{k} \neq \varnothing$ and $\forall n \geq k: \mathbb{T} \cap S_{n}=\varnothing$. Then $\mathbb{T}$ cannot intersect $S_{k}$ in only one component, so it has to come back through $S_{k}$. Thus, we have an annulus $A \subseteq \mathbb{T}$ that has both boundaries in $S_{k}$ and is contained in a submanifold of $M$ homeomorphic to $Y$. The annulus $A$ gives an isotopy between isotopic curves in $\partial Y$ and is therefore boundary parallel; see Remark 2.1. Hence, by an isotopy of $\mathbb{T}$ we can reduce $\left|\pi_{0}\left(\mathbb{T} \cap \bigcup_{i \in \mathbb{Z}} S_{i}\right)\right|$, contradicting the fact that it was minimal and nonzero. Therefore, $M$ is atoroidal.

Claim. The $M_{i, j}$ are hyperbolizable.

Proof of Claim. Since $M$ is atoroidal and for $i<j$ the $M_{i, j}$ are $\pi_{1}$-injective submanifolds, they are also atoroidal. Moreover, since the $M_{i, j}$ are compact manifolds with infinite $\pi_{1}$, they are hyperbolizable by Thurston's Hyperbolization Theorem Kap09.

The manifold $M$ is exhausted by the hyperbolizable $\pi_{1}$-injective submanifolds $M_{i} \doteq M_{-i, i}$.

Claim. The manifold $M$ is locally hyperbolic. 
Proof of Claim. To do so it suffices to show that given any finitely generated $H \leqslant$ $\pi_{1}(M)$ the cover $M(H)$ corresponding to $H$ factors through a cover $N \rightarrow M$ that is hyperbolizable. Let $\gamma_{1}, \ldots, \gamma_{n} \subseteq M$ be loops generating $H$. Since the $M_{i}$ exhaust $M$ we can find some $i \in \mathbb{N}$ such that $\left\{\gamma_{k}\right\}_{1 \leq k \leq n} \subseteq M_{i}$, hence the cover corresponding to $H$ factors through the cover induced by $\pi_{1}\left(M_{i}\right)$. We now want to show that the cover $M(i)$ of $M$ corresponding to $\pi_{1}\left(M_{i}\right)$ is hyperbolizable.

Since $\pi: M \rightarrow X$ is an infinite cyclic cover of $X$ we have that $M(i)$ is the same as the cover of $X$ corresponding to $\pi_{*}\left(\pi_{1}\left(M_{i}\right)\right)$. The resolution of the Tameness Ago98, CG06 and the Geometrization Theorems Per03b, Per03c, Per03a imply Simon's conjecture 3 that is, covers of compact irreducible 3-manifolds with finitely generated fundamental groups are tame Can08, Sim76. Therefore, since $X$ is compact by the Simon's Conjecture we have that $M(i)$ is tame. The submanifold $M_{i} \hookrightarrow M$ lifts homeomorphically to $\widetilde{M}_{i} \hookrightarrow M(i)$. By Whitehead's Theorem Hat02 the inclusion is a homotopy equivalence, hence $\widetilde{M}_{i}$ forms a Scott core for $M(i)$. Thus, since $\partial \widetilde{M}_{i}$ is incompressible and $M(i)$ is tame, we have that $M(i) \cong \operatorname{int}\left(M_{i}\right)$, and so it is hyperbolizable.

This concludes the proof.

Remark 2.4. Note that in the manifold $M$ the surfaces $S_{i}, S_{j}$ have no homotopic simple closed curve except for the loops $\gamma_{i} \doteq S_{i} \cap A$. If not, we would have an embedded cylinder $C$ not homotopic into $A_{i, j}$ which contradicts the fact that the characteristic submanifold of $M_{i, j}$ is given by a thickening of $A_{i, j}$. In particular, this gives us the important fact that for any homotopy equivalence $f: M \rightarrow N$ and any essential subsurface $F \subseteq S_{i}$ not isotopic to a neighbourhood of $\gamma$, we cannot homotope $f(F)$ through any $f\left(S_{j}\right)$ for $i \neq j$.

Lemma 2.5 (Homotopy equivalences). Given a tame 3-manifold $N$ let $\bar{N}$ be its compactification and let $g: M_{i, j} \rightarrow \bar{N}$ be a homotopy equivalence. Then, there exists a homotopy equivalence $f: M_{i, j} \rightarrow \bar{N}$ such that $f \simeq g$ and:

(1) $f\left(S_{k}\right)$ is embedded for all $i \leq k \leq j$;

(2) there are essential subsurfaces $T_{m}, T_{n}$ of $S_{m}, S_{n}$, respectively, whose components are homeomorphic to punctured tori, and where for all $i \leq m<$ $k<n \leq j$, the images $f\left(T_{n}\right), f\left(T_{m}\right)$ are separated in $N$ by $f\left(S_{k}\right)$. Moreover, the same holds for any surface $\Sigma_{k} \stackrel{\text { iso }}{\simeq} f\left(S_{k}\right)$ intersecting $f\left(S_{n}\right), f\left(T_{m}\right)$ minimally.

Proof. By Lemma 1.12 we get that $g: M_{i, j} \rightarrow \bar{N}$ is either homotopic to a homeomorphism $f$ or is given by a Dehn flip along the annulus $A_{i, j}$ of $M_{i, j}$. If $N$ is homeomorphic to $M_{i, j}$ we have nothing to do since the required map $f$ is the homeomorphism and (1) and (2) are true for $M_{i, j}$.

Therefore, we only need to deal with the case in which $f: M_{i, j} \rightarrow \bar{N}$ is a Dehn flip of $M$ along the annulus $A_{i, j}$. We will now explicitly write the Dehn flip $f$. Let $V \cong S^{1} \times I_{s} \times I_{t}$ be a regular neighbourhood of the annulus $A_{i, j}$ in $M_{i, j}$ such that $V \cap S_{k}, i \leq k \leq j$, are regular neighbourhoods $S^{1} \times\left\{s_{k}\right\} \times I_{t}$ of $\gamma$ in $S_{k}$. Similarly let $W \cong S^{1} \times I \times I$ be a regular neighbourhood of $A_{i, j}$ in $\bar{N}$. Let $F: V \rightarrow W$ be

\footnotetext{
${ }^{3}$ Final steps completed by Long and Reid, see Can08.
} 
given by

$$
F(x, s, t) \doteq\left\{\begin{array}{l}
(x, 2 t(1-s)+(1-2 t) s, t), \quad 0 \leq t \leq \frac{1}{2} \\
(x,(2-2 t)(1-s)+(2 t-1) s, t), \quad \frac{1}{2} \leq t \leq 1
\end{array}\right.
$$

and let $f: M_{i, j} \rightarrow \bar{N}$ be the homotopy equivalence obtained by extending $F$ via the homeomorphism of $\overline{M_{i, j} \backslash V} \rightarrow \bar{N} \backslash W$ coming from Lemma 1.12, Moreover, for $M_{i, j}^{\prime} \doteq \overline{M_{i, j} \backslash V}$ the homeomorphism $F$ is the identity on $V \cap M_{i, j}^{\prime}$. Then $f$ realises the Dehn flip from $M_{i, j}$ to $\bar{N}$. See Figure 3. The homeomorphism of $M_{i, j}^{\prime}$ preserves the order of the surfaces, it is the identity on $\partial V \cap M_{i, j}^{\prime}$, and hence for all $i \leq k \leq j$ the surfaces $f\left(S_{k}\right)$ are embedded. This concludes the proof of (1).

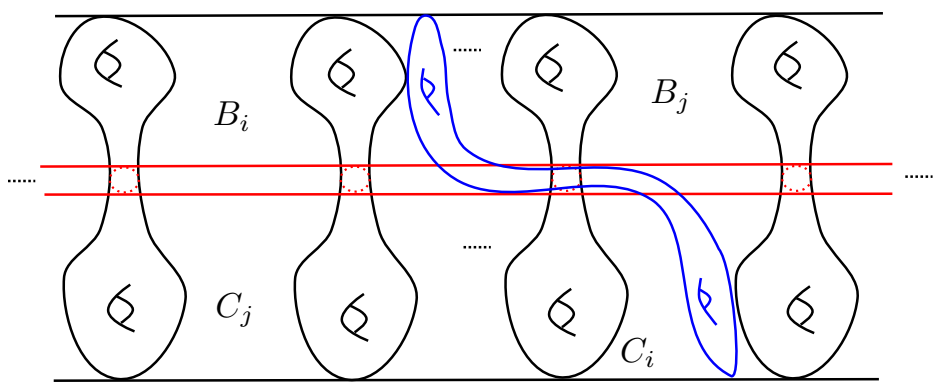

Figure 3. The surface in blue is an embedded push-off of $f\left(S_{i}\right)$ in $N \simeq \operatorname{int}\left(M_{i, j}\right)$ when $\bar{N}$ is a Dehn flip of $M_{i, j}$.

For (2) note that for all $i \leq k \leq j$ we have that $S_{k} \backslash V$ is given by two essential punctured tori $T_{k}^{ \pm}$. Moreover, for all $i \leq n \neq k \leq j$ we have that the essential tori $T_{n}^{ \pm}$are separated by $f\left(S_{k}\right)$. This again follows from the fact that $f$ is the identity on $\partial V \cap M_{i, j}^{\prime}$ and so it preserves ordering. Thus, we always see, up to isotopy, the following configuration:

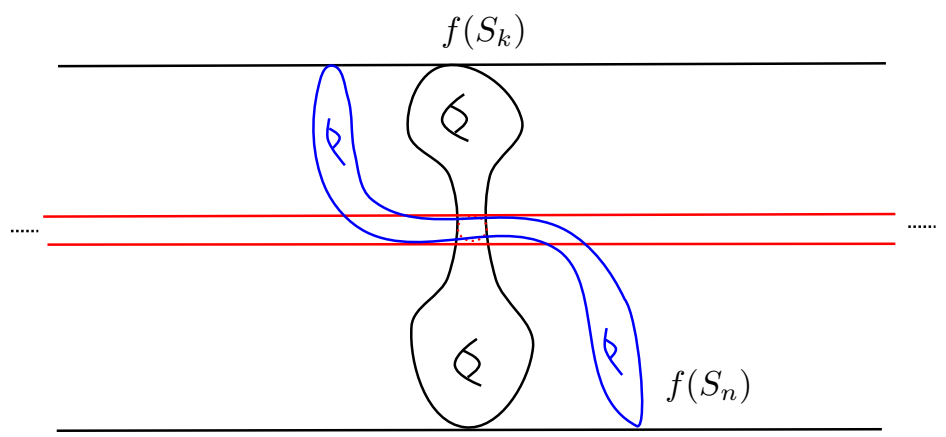


Finally, if $\Sigma_{k} \stackrel{\text { iso }}{\simeq} f\left(S_{k}\right)$ and intersects $f\left(S_{n}\right), f\left(S_{m}\right)$ minimally, we have that all components of intersections of $f\left(S_{n}\right), f\left(S_{m}\right)$, and $\Sigma_{k}$ are isotopic to the intersection of $f\left(S_{n}\right), f\left(S_{m}\right)$ with the annulus $A_{i, j}$. If the subsurfaces $f\left(T_{n}\right), f\left(T_{m}\right)$ are not separated by $\Sigma_{k}$, it means that they all lie in the same component $N_{1}$ of $N \backslash \Sigma_{k}$.

By the isotopy extension Theorem Hir94, 8.1.3] we have $\Sigma_{n}, \Sigma_{m}$ isotopic to $f\left(S_{n}\right)$ and $f\left(S_{m}\right)$, respectively, and subsurfaces $T_{n}^{\prime}, T_{m}^{\prime}$ isotopic to $f\left(T_{n}\right), f\left(T_{m}\right)$ that are separated in $N$ by $\Sigma_{k}$. Therefore, we can find a simple closed loop $\alpha$ in one of the essential subsurfaces $T_{n}^{\prime}, T_{m}^{\prime}$ that is not contained in $N_{1}$. Since we assumed that $f\left(T_{n}\right), f\left(T_{m}\right)$ are contained in $N_{1}$ the loop $\alpha$ is homotopic into $\Sigma_{k}$, contradicting Remark 2.4.

Definition 2.6. Given a hyperbolic 3-manifold $M$, a useful simplicial hyperbolic surface is a surface $S$ with a 1-vertex triangulation $\mathcal{T}$, a preferred edge $e$, and a map $f: S \rightarrow M$, such that:

(1) $f(e)$ is a geodesic in $M$;

(2) every edge of $\mathcal{T}$ is mapped to a geodesic segment in $M$;

(3) the restriction of $f$ to every face of $\mathcal{T}$ is a totally geodesic immersion.

By [Can96, Bon86] every $\pi_{1}$-injective map $f: S \rightarrow M$ with a 1-vertex triangulation with a preferred edge can be homotoped so that it becomes a useful simplicial surface. Moreover, with the path metric induced by $M$ a useful simplicial surface is negatively curved and the map becomes 1-Lipschitz.

Lemma 2.7. Let $N \cong \mathbb{H}^{3} / \Gamma$ be a hyperbolic 3-manifold homotopy equivalent to $M$. Then $\gamma$ is represented by a parabolic element in $\Gamma$.

Proof. Assume that $f_{*}(\gamma)$ is represented by a hyperbolic element, and let $A \subseteq M$ be the essential bi-infinite annulus obtained as the limit of the $A_{-i, i}$. Since all $S_{i}$ are incompressible in $M$ and $f$ is a homotopy equivalence, the maps $f: S_{i} \rightarrow N$ are $\pi_{1}$-injective. Let $\tau_{i}$ be 1-vertex triangulations of $S_{i}$ realising $\gamma_{i} \doteq S_{i} \cap A$ as an edge in the 1-skeleton. Then, by Can96, Bon86] we can realise the maps $f: S_{i} \rightarrow N$ by useful simplicial hyperbolic surfaces $\Sigma_{i} \subseteq N$ such that $\Sigma_{i} \simeq f\left(S_{i}\right)$ and the image of $\gamma_{i}$ is the unique geodesic representative $\bar{\gamma}$ of $f_{*}(\gamma)$.

In the simplicial hyperbolic surfaces $\Sigma_{i}$ a maximal one-sided collar neighbourhood of $\bar{\gamma}$ has area bounded by the total area of $\Sigma_{i}$. Since the simplicial hyperbolic surfaces are all genus two by Gauss-Bonnet, we have that $A\left(\Sigma_{i}\right) \leq 2 \pi\left|\chi\left(\Sigma_{i}\right)\right|=4 \pi$. Therefore, the radius of a one-sided collar neighbourhood is uniformly bounded by some constant $K=K\left(\chi\left(\Sigma_{i}\right), \ell_{N}(\bar{\gamma})\right)<\infty$ for $\ell_{N}(\bar{\gamma})$ the hyperbolic length of $\gamma$ in $N$. Then for $\xi>0$ in the simplicial hyperbolic surface $\Sigma_{i}$ the $K+\xi$ two-sided neighbourhood of $\bar{\gamma}$ is not embedded and contains an essential 4-punctured sphere $X_{i} \subseteq \Sigma_{i}$. Since simplicial hyperbolic surfaces are 1-Lipschitz, the 4-punctured sphere is contained in a $K+\xi$ neighbourhood $C$ of $\bar{\gamma}$ in $N$. The curves $\alpha_{i}$ obtained by joining the seams of the pants decomposition of $X_{i}$ induced by $\gamma_{i}$ have length bounded by $L \doteq 2 K+2 \xi+\ell_{N}(\bar{\gamma})$. Since there are infinitely many $\alpha_{i}$ and they are all homotopically distinct, we have that $\Gamma$ is not discrete since the $\alpha_{i}$ move a lift of $\bar{\gamma}$ a uniformly bounded amount.

Proposition 2.8. Let $f: M \rightarrow N$ be a homotopy equivalence. Then for all $i$ the maps: $f: S_{i} \rightarrow N$ have embedded representatives $\Sigma_{i} \simeq f\left(S_{i}\right)$ in $N$. 
Proof. Fix a triangulation $\tau$ of $N$. Since the $f\left(S_{i}\right)$ are $\pi_{1}$-injective by taking a refinement $\tau_{i}$ of $\tau$ outside a precompact neighbourhood $U_{i}$ of $f\left(S_{i}\right)$, we can homotope $f\left(S_{i}\right)$ to be a PL-least area surface $\Sigma_{i}$ with respect to a weight system induced by $\tau_{i}$; see FHS83, Kap09 JR88. We now want to show that they are embedded. To do so, by FHS83, Kap09, JR88, it suffices to show that there is a neighbourhood $\Sigma_{i} \subseteq U_{i}$ lifting homeomorphically to a cover $N$ of $M$ in which $\Sigma_{i}$ has an embedded representative.

Let $U_{i} \subseteq N$ be a neighbourhood of $\Sigma_{i}$ in $N$. Since $\pi_{1}\left(f_{*}\left(M_{j}\right)\right)$ exhaust $\pi_{1}(N)$ there is $j=j(i)$ such that $\pi_{1}\left(U_{i}\right) \subseteq \pi_{1}\left(f_{*}\left(M_{j}\right)\right)$. Let $p_{j}: N_{j} \rightarrow N$ denote the cover of $N$ corresponding to $\pi_{1}\left(f_{*}\left(M_{j}\right)\right)$. Then, we have the following commutative diagram:

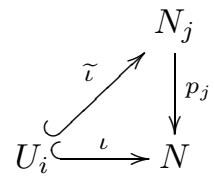

in which $p_{j}$ homeomorphically maps $\widetilde{\iota}\left(U_{i}\right)$ to $U_{i}$. Moreover, let $\widetilde{\tau}_{i}$ be the triangulation of $N_{j}$ induced by $\tau_{i}$, and let $\widetilde{\Sigma}_{i} \subseteq \widetilde{\iota}\left(U_{i}\right)$ be the homeomorphic lift of $\Sigma_{i}$ under $p_{j}$. The surface $\widetilde{\Sigma}_{i}$ is then a PL-least area surface for $\widetilde{\tau}_{i}$. Thus, if $\widetilde{\Sigma}_{i}$ is embedded we are done.

By Lemma 2.3 the homotopy equivalence $\left.\widetilde{f}\right|_{M_{j}}: M_{j} \rightarrow N_{j}$ is homotopic to a homeomorphism. Therefore $\widetilde{\Sigma}_{i}$ has an embedded representative in $N_{j}$, and hence by FHS83, Kap09, JR88, we have that $\widetilde{\Sigma}_{i}$ is embedded as well.

We will now prove the Theorem which we now restate.

Theorem 2.9. The manifold $M$ is locally hyperbolic and without divisible elements in $\pi_{1}(M)$ but is not homotopy equivalent to any hyperbolic 3-manifold.

Proof. By Lemmas 2.2 and 2.3 we only need to show that $M$ is not homotopy equivalent to any hyperbolic 3 -manifold $N$. The proof will be by contradiction. Assume that we have a homotopy equivalence $f: M \rightarrow N$ for $N \cong \mathbb{H}^{3} / \Gamma$ a hyperbolic 3-manifold. By Lemma 2.7 we have that for $\gamma$ the element of $\pi_{1}(M)$ generating the fundamental group of the bi-infinite essential annulus $A \subseteq M f_{*}(\gamma)$ is represented by a parabolic element in $\Gamma$ (with an abuse of notation we will refer to this element by $\gamma$ as well). Thus, in $N$ we have a cusp $E \doteq E_{\gamma}$ corresponding to $\gamma$. Moreover, Proposition 2.8 gives us a collection $\left\{\Sigma_{i}\right\}_{i \in \mathbb{Z}}$ of embedded genus two surfaces contained in neighbourhoods $U_{i}$ of $f\left(S_{i}\right)$ in $N$ such that $\Sigma_{i} \simeq f\left(S_{i}\right)$. Moreover the $\Sigma_{i}$ 's are incompressible and separating in $N$. The fact that they are separating follows from $f_{*}$ being an isomorphism in homology and the fact that the $S_{i}$ 's are not dual to 1-cycles in $M$. Similarly, they are incompressible since the $S_{i}$ are and $f$ is a homotopy equivalence. Therefore, if we take the surface $\Sigma_{0}$ we have that $N \mid \Sigma_{0} \doteq N \backslash \operatorname{int}\left(N_{r}\left(\Sigma_{0}\right)\right)$ is given by two manifolds $N_{1}, N_{2}$ with boundary a surface isotopic in $N$ to $\Sigma_{0}$.

The element $\gamma \in \Gamma$ is parabolic with cusp $E$, and each $\Sigma_{i}$ has a simple closed loop $\gamma_{i}$ homotopic in $N$ to $\gamma$ such that $\overline{\Sigma_{i} \backslash N_{r}\left(\gamma_{i}\right)}$ is given by two punctured tori $T_{i}^{ \pm}$with boundary isotopic to $\gamma_{i}$. Without loss of generality we can assume that $E \subseteq N_{2}$. Moreover, up to an isotopy of each $\Sigma_{i}$ we can also assume that for all $i \in \mathbb{Z}$ the surfaces $\Sigma_{i}$ are transverse to $\Sigma_{0}$ and that $\left|\pi_{0}\left(\Sigma_{i} \cap \Sigma_{0}\right)\right|$ is minimal. 
Claim. Every component $\alpha \in \pi_{0}\left(\Sigma_{i} \cap \Sigma_{0}\right)$ is isotopic to $\gamma_{i}$ and $\gamma_{0}$.

Proof of Claim. Since $\Sigma_{i}, \Sigma_{0}$ are incompressible and we minimised $\Sigma_{i} \cap \Sigma_{0}$, we have that every $\alpha \in \pi_{0}\left(\Sigma_{i} \cap \Sigma_{0}\right)$ has to be essential in both surfaces. By Remark 2.4 we have that the only simple closed curve in $S_{i}$ homotopic into $S_{0}$ is $\gamma_{i}$, which is homotopic to $\gamma_{0}$.

Thus in $N$ we have that the punctured tori $T_{i}^{ \pm}$to $\Sigma_{i}$ are either on the same side of $\Sigma_{0}$ or on opposite sides as in Figure 4 .
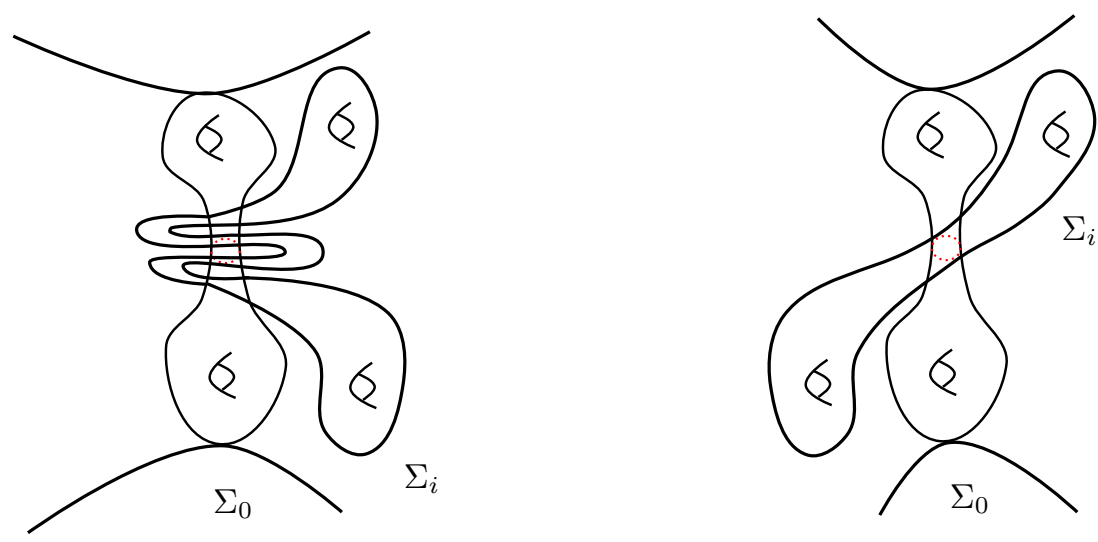

Figure 4. Possible configurations of the embedded surfaces $\Sigma_{0}, \Sigma_{i}$ in $N$.

Moreover, all the components of $\Sigma_{i} \cap \Sigma_{0}$ are contained in neighbourhoods of $\gamma_{0}$ and $\gamma_{i}$.

Claim. There are infinitely many punctured tori $\left\{T_{n}\right\}_{n \in \mathbb{N}} \subseteq N_{1}$ such that $T_{n}$ is a component of $T_{i_{n}}^{ \pm}$.

Proof of Claim. Consider $\Sigma_{-i}, \Sigma_{0}, \Sigma_{i}$, and a cover $\pi_{j}: N_{-i, i} \rightarrow N$ corresponding to the subgroup $f_{*}\left(\pi_{1}\left(M_{-j, j}\right)\right) \subseteq \pi_{1}(N)$ where $\Sigma_{0} \cup \Sigma_{i} \cup \Sigma_{-i}$ lifts homeomorphically. Assume that there only finitely many $T_{i}^{ \pm}$that are contained in $N_{1}$. Then, for infinitely many $T_{i}^{ \pm}$in the covers $N_{j}$ we see the configuration in Figure 5 .

Let $g$ be as in Lemma 2.5 and homotopic to the homotopy equivalence $\tilde{f}$ : $M_{-j, j} \rightarrow \bar{N}_{-j, j}$. Since $\widetilde{\Sigma}_{k}$ and $g\left(S_{k}\right)$ are incompressible closed surfaces by Wal68] we have that $\widetilde{\Sigma}_{k} \simeq g\left(S_{k}\right)$, and by (2) of Lemma 2.5 we have that $\widetilde{\Sigma}_{k}$ separates the punctured tori $g\left(Q_{i}^{ \pm}\right), g\left(Q_{-i}^{ \pm}\right)$in $g\left(S_{i}\right)$ and $g\left(S_{-i}\right)$. Thus, we have a punctured torus, say $f\left(Q_{i}^{+}\right)$, that is contained in $\widetilde{N}_{1}$ and such that the corresponding punctured torus $\widetilde{T}_{i}^{+} \subseteq \widetilde{\Sigma}_{i}$ is contained in $\widetilde{N}_{2}$. Let $\alpha \subseteq T_{i}^{+}$be any essential nonperipheral curve. Then since $\alpha$ is homotopic into $f\left(\bar{Q}_{i}^{+}\right)$and $\widetilde{\Sigma}_{0}$ separates, we have that 

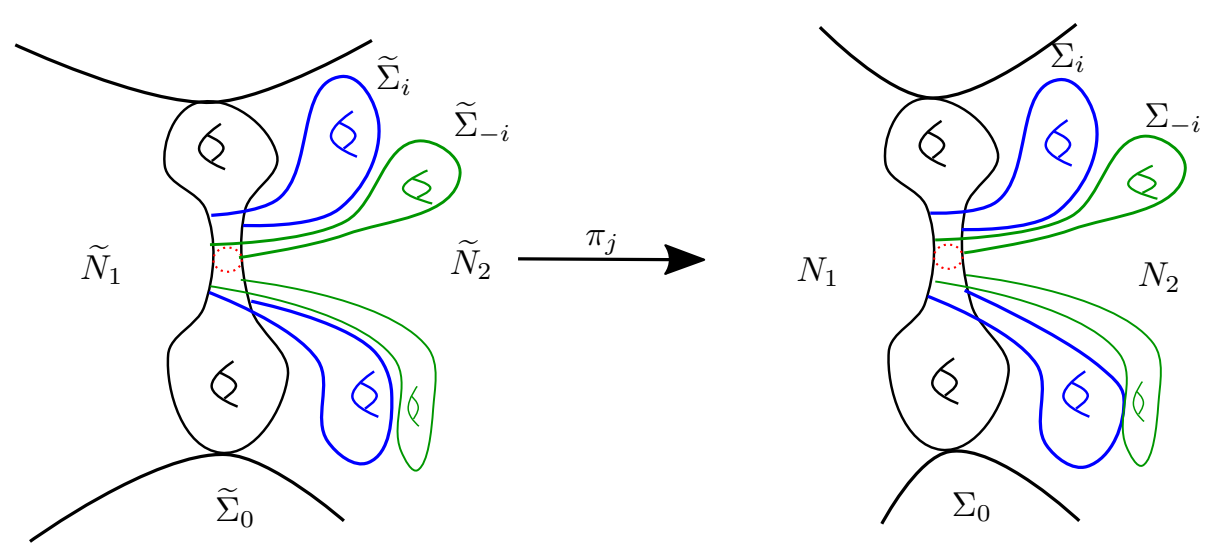

Figure 5 . The tori $T_{i}^{ \pm}$are marked by the surfaces $\Sigma_{i}$, to which they are subsurfaces.

$\alpha$ is homotopic into $\widetilde{\Sigma}_{0}$, contradicting Remark 2.4 Therefore, we have infinitely many punctured tori $\left\{T_{n}\right\}_{n \in \mathbb{N}}$ with boundary $\partial T_{n} \doteq \gamma_{n}$ isotopic to $\gamma$ such that $\forall n: T_{n} \subseteq N_{1}$.

We can now reach a contradiction with the fact that $\Gamma$ is a discrete group. Let $\mu \doteq \operatorname{inj}_{N}\left(\Sigma_{0}\right)$, and let $\varepsilon \doteq \min \left\{\mu, \mu_{3}\right\}$ where $\mu_{3}$ is the 3 -dimensional Margulis constant (see [BP92]). Since the $T_{n}$ 's are $\pi_{1}$-injective by picking a 1-vertex triangulation $\tau_{n}$ with preferred edge corresponding to $\gamma_{n}$, we can realise the $T_{n}$ 's by useful simplicial hyperbolic surfaces $P_{n}$.

The surfaces $P_{n}$ are mapping $\gamma_{n}$ to the cusps $E$ and cannot be homotoped through $\Sigma_{0}$ since again we would contradict Remark 2.4. Hence, we get that for all $n, P_{n} \cap \Sigma_{0} \neq \varnothing$. Let $x_{n} \in P_{n} \cap \Sigma_{n}$; then $\operatorname{inj}_{x_{n}}\left(P_{n}\right) \geq \varepsilon$. Since the surfaces $P_{n}$ are negatively curved by the Bounded Diameter Lemma Bon86, Thu78a, we get that we can find loops $\alpha_{n}, \beta_{n} \in \pi_{1}\left(P_{n}, x_{n}\right)$ whose length is bounded by $\frac{8}{\varepsilon}$ and such that they generate a rank two free group. Since $\left\langle\alpha_{n}, \beta_{n}\right\rangle \cong \mathbb{F}_{2}$ we have that at least one of $\alpha_{n}, \beta_{n}$ is not homotopic to $\gamma_{n}$. Without loss of generality we can assume that this element is always $\alpha_{n}$. By Remark 2.4 the collection $\left\{\alpha_{n}\right\}_{n \in \mathbb{N}}$ are all distinct elements in $\Gamma$. Moreover, we have that $\ell_{N}\left(\alpha_{n}\right) \leq \frac{8}{\varepsilon}$. Let $D \doteq \operatorname{diam}\left(\Sigma_{0}\right)$, pick $x \in \Sigma_{0}$, and fix $\widetilde{x}$ to be a lift in $\widetilde{\Sigma}_{0} \subseteq \mathbb{H}^{3}$. Then for lifts $\widetilde{x}_{n}$ of $x_{n}$ we have that

$$
\begin{aligned}
d_{\mathbb{H}^{3}}\left(\alpha_{n}(\widetilde{x}), \widetilde{x}\right) & \leq d_{\mathbb{H}^{3}}\left(\alpha_{n}(\widetilde{x}), \alpha_{n}\left(\widetilde{x}_{n}\right)\right)+d_{\mathbb{H}^{3}}\left(\alpha_{n}\left(\widetilde{x}_{n}\right), \widetilde{x}_{n}\right)+d_{\mathbb{H}^{3}}\left(\widetilde{x}_{n}, \widetilde{x}\right) \\
& \leq D+\frac{8}{\varepsilon}+D \\
& =2 D+\frac{8}{\varepsilon} .
\end{aligned}
$$

Thus the family $\left\{\alpha_{n}\right\}_{n \in \mathbb{N}}$ has an accumulation point in $\mathrm{PSL}_{2}(\mathbb{C})$ contradicting the discreteness of $\Gamma$. 


\section{ACKNOWLEDGMENTS}

I would like to thank Ian Biringer and Martin Bridgeman for many helpful discussions. Moreover, I would like to thank the anonymous referee for corrections.

\section{REFERENCES}

[Ago98] Ian Agol, Topology of hyperbolic 3-manifolds, ProQuest LLC, Ann Arbor, MI, 1998. Thesis (Ph.D.)-University of California, San Diego. MR.2698165

[Bon86] Francis Bonahon, Bouts des variétés hyperboliques de dimension 3 (French), Ann. of Math. (2) 124 (1986), no. 1, 71-158, DOI 10.2307/1971388. MR847953

[BP92] Riccardo Benedetti and Carlo Petronio, Lectures on hyperbolic geometry, Universitext, Springer-Verlag, Berlin, 1992. MR 1219310

[Can96] Richard D. Canary, A covering theorem for hyperbolic 3-manifolds and its applications, Topology 35 (1996), no. 3, 751-778, DOI 10.1016/0040-9383(94)00055-7. MR1396777

[Can08] Richard D. Canary, Marden's tameness conjecture: history and applications, Geometry, analysis and topology of discrete groups, Adv. Lect. Math. (ALM), vol. 6, Int. Press, Somerville, MA, 2008, pp. 137-162. MR2464394

[CG06] Danny Calegari and David Gabai, Shrinkwrapping and the taming of hyperbolic 3manifolds, J. Amer. Math. Soc. 19 (2006), no. 2, 385-446, DOI 10.1090/S0894-034705-00513-8. MR2188131

[CM04] Richard D. Canary and Darryl McCullough, Homotopy equivalences of 3-manifolds and deformation theory of Kleinian groups, Mem. Amer. Math. Soc. 172 (2004), no. 812, xii+218, DOI 10.1090/memo/0812. MR2096234

[Cre17] Tommaso Cremaschi, Hyperbolization on infinite type 3-manifolds, arXiv:1904.11359 (2017).

[DHM06] Kelly Delp, Diane Hoffoss, and Jason Fox Manning, Problems in groups, geometry, and three-manifolds. http://arxiv.org/pdf/1512.04620.pdf, 2006.

[FHS83] Michael Freedman, Joel Hass, and Peter Scott, Least area incompressible surfaces in 3-manifolds, Invent. Math. 71 (1983), no. 3, 609-642, DOI 10.1007/BF02095997. MR695910

[Hat02] Allen Hatcher, Algebraic topology, Cambridge University Press, Cambridge, 2002. MR 1867354

[Hat07] Allen Hatcher, Basic notes on 3-manifolds. http://www.math.cornell.edu/ hatcher/ 3M/3Mfds.pdf, 2007.

[Hem76] John Hempel, 3-Manifolds, Princeton University Press, Princeton, N. J.; University of Tokyo Press, Tokyo, 1976. Ann. of Math. Studies, No. 86. MR0415619

[Hir94] Morris W. Hirsch, Differential topology, Graduate Texts in Mathematics, vol. 33, Springer-Verlag, New York, 1994. Corrected reprint of the 1976 original. MR1336822

[Jac80] William Jaco, Lectures on three-manifold topology, CBMS Regional Conference Series in Mathematics, vol. 43, American Mathematical Society, Providence, R.I., 1980. MR.565450

[Joh79] Klaus Johannson, Homotopy equivalences of 3-manifolds with boundaries, Lecture Notes in Mathematics, vol. 761, Springer, Berlin, 1979. MR551744

[JR88] William Jaco and J. Hyam Rubinstein, PL minimal surfaces in 3-manifolds, J. Differential Geom. 27 (1988), no. 3, 493-524. MR.940116

[JS78] William Jaco and Peter B. Shalen, A new decomposition theorem for irreducible sufficiently-large 3-manifolds, Algebraic and geometric topology (Proc. Sympos. Pure Math., Stanford Univ., Stanford, Calif., 1976), Proc. Sympos. Pure Math., XXXII, Amer. Math. Soc., Providence, R.I., 1978, pp. 71-84. MR520524

[Kap09] Michael Kapovich, Hyperbolic manifolds and discrete groups, Modern Birkhäuser Classics, Birkhäuser Boston, Ltd., Boston, MA, 2009. Reprint of the 2001 edition. MR 2553578

[Mar07] A. Marden, Outer circles: An introduction to hyperbolic 3-manifolds, Cambridge University Press, Cambridge, 2007. MR2355387

[Per03a] Grigori Perelman, Finite extinction time for the solutions to the Ricci flow on certain three-manifolds. https://arxiv.org/pdf/math/0307245.pdf, 2003. 
[Per03b] Grigori Perelman, Ricci flow with surgery on three-manifolds. https://arxiv.org/abs/ math/0303109, 2003.

[Per03c] Grigori Perelman, The entropy formula for the Ricci flow and its geometric applications. https://arxiv.org/abs/math/0211159, 2003.

[RS90] J. H. Rubinstein and G. A. Swarup, On Scott's core theorem, Bull. London Math. Soc. 22 (1990), no. 5, 495-498, DOI 10.1112/blms/22.5.495. MR.1082023

[Sco73] G. P. Scott, Compact submanifolds of 3-manifolds, J. London Math. Soc. (2) 7 (1973), 246-250, DOI 10.1112/jlms/s2-7.2.246. MR326737

[HS96] Luke Harris and Peter Scott, The uniqueness of compact cores for 3-manifolds, Pacific J. Math. 172 (1996), no. 1, 139-150. MR1379290

[Sha75] Peter B. Shalen, Infinitely divisible elements in 3-manifold groups, Knots, groups, and 3-manifolds (Papers dedicated to the memory of R. H. Fox), Ann. of Math. Studies, No. 84, Princeton Univ. Press, Princeton, N.J., 1975, pp. 293-335. MR0375280

[Sim76] Jonathan Simon, Compactification of covering spaces of compact 3-manifolds, Michigan Math. J. 23 (1976), no. 3, 245-256 (1977). MR 431176

[Thu78a] William B. Thurston, Geometry and Topology of 3-manifolds. Princeton Mathematics Department Lecture Notes. http://library.msri.org/books/gt3m/.

[Thu97] William P. Thurston, Three-dimensional geometry and topology. Vol. 1, Princeton Mathematical Series, vol. 35, Princeton University Press, Princeton, NJ, 1997. Edited by Silvio Levy. MR1435975

[Thu82] William P. Thurston, Three-dimensional manifolds, Kleinian groups and hyperbolic geometry, Bull. Amer. Math. Soc. (N.S.) 6 (1982), no. 3, 357-381, DOI 10.1090/S02730979-1982-15003-0. MR648524

[Wal68] Friedhelm Waldhausen, On irreducible 3-manifolds which are sufficiently large, Ann. of Math. (2) 87 (1968), 56-88, DOI 10.2307/1970594. MR224099

Department of Mathematics, University of Southern California, 140 Commonwealth Avenue, Chestnut Hill, Massachusetts 02467

Email address: cremasch@usc.edu 